\title{
Evaluation of Oxidative Stress Parameters in Liver in Pentylenetetrazole - Induced Acute and Chronic Epilepsy Model in Rats
}

\author{
Aktas Ahmet*1 and Sahin Bilal ${ }^{2}$ \\ ${ }^{1}$ Department of Internal Medicine, Faculty of Medicine, Cumhuriyet University, Turkey \\ ${ }^{2}$ Department of Physiology, Faculty of Medicine, Cumhuriyet University, Turkey \\ *Corresponding author: Aktas Ahmet, Department of Internal Medicine, Faculty of Medicine, Cumhuriyet University, Sivas, Turkey. \\ To Cite This Article: Aktas Ahmet. Evaluation of Oxidative Stress Parameters in Liver in Pentylenetetrazole - Induced Acute and Chronic Epilepsy \\ Model in Rats. Am J Biomed Sci \& Res. 2019 - 5(6). AJBSR.MS.ID.000966. DOI: 10.34297/AJBSR.2019.05.000966.
}

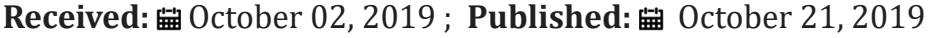

\begin{abstract}
Epilepsy occurs due to periodic and spontaneous excessive electrical discharges of cerebral, cortical and subcortical neurons. Epilepsy affects about $1 \%$ of the global world population. In epilepsy, the formation of reactive oxygen species (ROS) in the brain usually increases with recurrent seizures. The aim of this study was to evaluate the levels of oxidative damage in the chronic and acute epilepsy model by measuring AST and ALT enzyme levels from serum samples and CAT, SOD and TBARS levels in liver tissues. In our study we found that, serum AST levels of acute epileptic model group were found to be significantly higher than control and chronic epileptic model group (p<0.05). SOD levels of chronic epileptic model group were found to be significantly higher than acute epileptic model group and control groups (p<0.05). CAT levels of chronic epileptic model group were found to be significantly lower than control group $(\mathrm{p}<0.05)$.
\end{abstract}

Keywords: Epilepsy; Oxidative stress; Liver enzymes

\section{Introduction}

Epilepsy occurs due to periodic and spontaneous excessive electrical discharges of cerebral, cortical and subcortical neurons. Epilepsy affects about $1 \%$ of the global world population $[1,2]$. Pentylenetetrazole (PTZ) is a GABA receptor blocker from chemical tetrazole. It is often used to model the occurrence of animal epilepsy and evaluate the efficacy of antiepileptic agents. It is one of the drugs commonly used in the formation of generalized tonic-clonic epileptic seizures [3]. In generalized epilepsy, the formation of reactive oxygen species (ROS) in the brain increases with recurrent seizures [4]. Increased oxidative stress due to increased free radical release has been associated with underlying pathogenesis in the onset and development of epileptic seizures. Therefore, it has been concluded that antioxidant treatment may provide neuroprotective effect by reducing oxidative stress in epilepsy treatment [5]. Increased oxidative stress in the central nervous system has been shown to increase in various experimental epilepsy models and electroshock models [6] such as the amygdala burning model [7] kainic acid model [8], PTZ model [9], sound stimulation (switching) model [10]. The liver is an organ that regulates different functions such as secretion, metabolism, detoxification and storage in the body and is sensitive to oxidative damage $[11,12]$. The aim of this study was to evaluate the levels of oxidative injury in chronic and acute epilepsy model by measuring AST and ALT enzyme levels from serum samples and CAD, TBARS and SOD levels in liver tissues.

\section{Materials and Methods}

\section{Subjects}

Male, adult 200-250 g Wistar rats $(\mathrm{n}=18)$ were used in the experiment. Ethics approval was obtained from Sivas Cumhuriyet University Faculty of Medicine Ethics Committee. Animals were divided into six groups:

a) Control Group (Control; $\mathrm{n}=6$ ); rats, single dose i.p. saline,

b) Acute Epileptic Model Group $(n=6)$; rats, single dose i.p. PTZ (45 mg / kg),

c) Chronic Epileptic Model Group (n = 6); rats, repeated i.p. doses PTZ (35 mg / kg) every other day for 15(fifteen) times. 


\section{Seizure Model}

The chronic epilepsy model is induced by 15 injections of 35 $\mathrm{mg} / \mathrm{kg}$ PTZ and the acute epilepsy model is induced by single dose PTZ (45 mg / kg). Rats were observed for epileptic seizures for 30 minutes after PTZ injection. The activity was performed as general epileptic seizures beginning with clonus of the forefoot and facial muscles and continuing with tail and neck extensions, tonic flexionextension and loss of straightening reflex and generally extended clonic activity. Transport times and behavioral characteristics of epileptic activities were recorded. Animals were killed by guillotine 24 hours after saline administration or PTZ-induced seizures (single or final seizure).

\section{Tissue Assessment and Methods}

Blood was collected before sacrifice from the animals and centrifuged at $2000 \mathrm{rpm}$ for 20 minutes. Serum samples were taken. AST and ALT levels were measured from serum samples using automatic analyzer in Sivas Cumhuriyet University Hospital. After the blood collection, the animals were sacrificed, and the liver tissues of the animals were removed for sampling. It was placed in PBS, which was five times higher than the extracted liver tissue. A manual homogenizer was used to prevent tissue degeneration and all extracted tissues were homogenized on ice in this PBS. Samples were centrifuged at $3000 \mathrm{rpm}$ for 20 minutes and the supernatants were separated. SOD, CAT and TBARS levels in liver supernatants were measured using sandwich-ELISA method according to the manufacturer's protocol and protein concentration was determined by Bradford protein assay kit.

\section{Statistics}

The mean \pm SD was used for all data. The SPSS statistical package (SPSS Inc., Chicago, IL) was used to perform statistical analyzes in our study. Statistical analysis was performed to compare changes between individual groups using variance analysis (ANOVA) followed by post ANOVA (Tukey's HSD) test. The $p$ value was accepted as $<0.05$ to accept statistically significant difference between the groups.

\section{Results}

Table 1 includes serum AST and ALT levels and Chart 1 includes SOD, TBARS and CAT levels in liver tissue in all (3) groups. There was no significant difference between the groups in terms of serum ALT and liver TBARS parameters ( $p>0.05$ ). There was no significant difference between chronic epileptic model group and control group ( $p>0.05$ ). Serum AST levels of the chronic epileptic model and control group were significantly lower than the acute epileptic model group ( $p<0.05)$. SOD levels of the chronic epileptic model group were significantly higher than the acute epileptic model group and the control group ( $p<0.05)$. CAT levels of the control group were significantly lower than those of the chronic epileptic model group $(\mathrm{p}<0.05)$ (Table 1) (Figure 1).

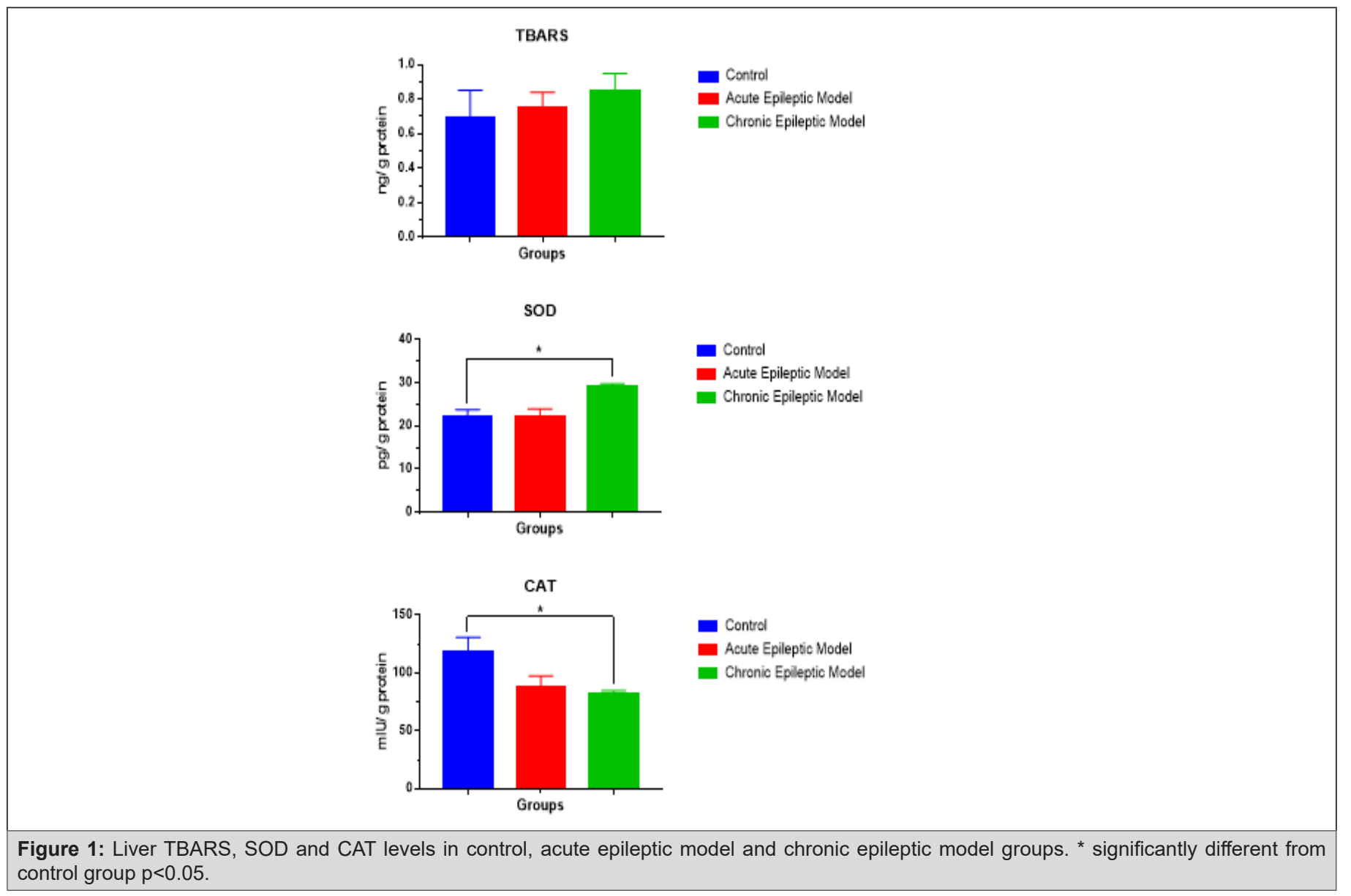


Table 1: Serum AST and ALT levels in control, acute epileptic model and chronic epileptic model groups.

\begin{tabular}{|c|c|c|}
\hline Groups & AST (U/L) & ALT (U/L) \\
\hline Control (n=6) & $114,70 \pm 8,48$ & $46,13 \pm 2,88$ \\
\hline $\begin{array}{c}\text { Acute epileptic model } \\
\text { (n=6) }\end{array}$ & $197,11 \pm 14,08^{*}$ & $45,01 \pm 3,68$ \\
\hline $\begin{array}{c}\text { Chronic epileptic } \\
\text { model (n=6) }\end{array}$ & $112,07 \pm 3,00$ & $42,30 \pm 2,58$ \\
\hline
\end{tabular}

*Significantly different from control group, $\mathrm{p}<0.05$

\section{Discussion}

Lipid peroxidation (MDA) is caused by free radicals such as NO. Furthermore, glutamine synthase can be directly inactivated for epileptic seizure activation, thereby causing abnormal accumulation of glutamate, the main stimulatory neurotransmitter $[13,3]$. In previous studies, it is thought that oxidative stress is an important cause of excitotoxicity and glutamate receptor activation [14]. SOD is one of the important antioxidants against free radicals. In addition, SOD is thought to prevent lipid peroxidation. Normally, lipid peroxidation occurs at low levels in each person and is the result of increased oxidative stress. MDA, the product of lipid peroxidation, has toxic effects on cell membranes and cells [11]. PTZ-induced epileptic seizures are associated with oxidative stress. Because it is a clinical epilepsy model that simulates actual epileptic seizures, it is widely used to create experimental epileptic models [15]. Akbas et al. [16] reported that increased oxidative stress caused by lipid peroxidation in hepatocytes during longterm epileptic seizures may lead to hepatocyte damage [16]. They used an epileptic model PTZ dose. They found that SOD and GSH in the liver and blood were significantly increased in the epileptic model group compared to the control group. We found that SOD levels were significantly lower in the acute epileptic model group and in the control, group compared to the chronic epileptic group ( $p<0.05)$. Activation of the nuclear factor erythroid 2-related factor (Nrf2) $[17,18]$ increases antioxidant defenses in brain cells, and there are mechanisms to prevent the occurrence of nitrogen species and reactive oxygen species. Nrf2 enhances the expression of various endogenous antioxidant enzymes and thus plays a key role in intracellular signaling and redox balance. The regulated ARE genes are activated in astrocytes. As a result, neurons have antioxidant defenses and detoxification. Thus, they protect the cells against oxidative stress [19]. In PTZ-induced animals, an increase in the expression of Nrf2 was observed (data in print), and a marked increase in immunoactivity against enzymes regulated by this factor suggests activation in epileptic seizure models [20]. SOD (CuZnSOD and MnSOD) found in cytosol and mitochondria are found in the antioxidant enzyme systems regulated by Nrf2. H2O can be eliminated by CAT in mitochondria and peroxisomes or neutralized in H2O with GPx in the cytosol [21]; in this paper CAT levels of the control group were significantly lower than those of the chronic epileptic model group $(p<0.05)$.
In previous studies, TBARS, the product of lipid oxidation, was used as an indicator of oxidative stress [22]. In our study there was no significant difference between the groups in terms of serum ALT and liver TBARS parameters ( $p>0.05$ ). There was no significant difference between chronic epileptic model group and control group ( $\mathrm{p}>0.05)$.

\section{Conclusion}

In conclusion, it causes increased oxidative damage and lipid peroxidation in PTZ-induced recurrent and single epileptic seizure models. In addition, antioxidant defense mechanisms have been decreased in these models. Increased oxidative stress, either recurrent or in a single epileptic seizure, causes damage to hepatocytes.

\section{References}

1. Yaari Y, Beck H (2002) "Epileptic neurons" in temporal lobe epilepsy. Brain pathology 12(2): 234-239.

2. (2001) Organization WH. The World Health Report 2001: Mental health: new understanding, new hope: World Health Organization Geneva, Switzerland Pp.100.

3. Ziylan YZ, Ates N (1989) Age-relatedchanges in regionalpattern of blood-brain barrier break down during epileptiform seizuresinduced by pentylenetetrazol. Neuroscience Letters 96(2): 179-184.

4. K Sudha, AV Rao, A Rao (2001) Oxidative stress and antioxidants in epilepsy. Clin Chim Acta 303(1-2): 19-24.

5. Shin EJ, Jeong JH, Chung YH, Kim WK, Ko KH, et al. (2011) Role of oxidative stres in epileptic seizures. Neurochem Int 59(2): 122-137.

6. T Barichello, F Bonatto, FR Agostinho, A Reinke, JC Moreira, et al. (2004) Structure-related oxidative damage in rat brain after acute and chronic electroshock. Neurochem Res 29(9): 1749-1753.

7. MV Frantseva, JL Perez Velazquez, G Tsoraklidis, AJ Mendonca, Y Adamchik, et al. (2000) Oxidative stress is involved in seizureinduced neurodegeneration in the kindling model of epilepsy. Neuroscience 97(3): 431-435.

8. MR Gluck, E Jayatilleke, S Shaw, AJ Rowan, V Haroutunian (2000) CNS oxidative stress associated with the kainic acid rodent model of experimental epilepsy. Epilepsy Res 39 (1): 63-71.

9. YK Gupta, MH Veerendra Kumar, AK Srivastava (2003) Effect of Centella asiatica on pentylenetetrazole-induced kindling, cognition and oxidative stress in rats. Pharmacol Biochem Behav 74(3): 579-585.

10. LV Vinogradova (2004) Audiogenic kindling in WAG/Rij rats: change in behavioral and electrophysiological responses to repetitive short acoustic stimulation. Zh Vyssh Nerv Deiat Im I P Pavlova 54(5): 638647.

11. N Seyhan, AG Canseven (2006) In vivo effects of ELF MFs on collagen synthesis, free radical processes, natural antioxidant system, respiratory burst system, immune system activities, and electrolytes in the skin, plasma, spleen, lung, kidney, and brain tissues. Electromagn Biol Med 25(4): 291-305.

12. Guyton JE, Hall A (2016) Guyton and Hall Texbook of Medical Physiology. Philadelphia, Pennsylvania 13: 881-884.

13. CN Oliver, PE Starke Reed, ER Stadtman, GJ Liu, JM Carney, RA Floyd (1990) Floyd Oxidative damage to brain proteins, loss of glutamine synthetase activity, and production of free radicals during ischemia/ reperfusion-induced injury to gerbil brain Proc Natl Acad Sci U S A 87(13): 5144-5147. 
14. M Patel (1996) Superoxide involvement in excitotoxicity: a SODmimetic holds promise as a novel neuroprotective agent. Mol Psychiatry 1(5): 362-363.

15. D Sahin, G Ilbay, N Ates (2003) Changes in the blood-brain barrier permeability and in the brain tissue trace element concentrations after single and repeated pentylenetetrazole-induced seizures in rats. Pharmacol Res 48(1):69-73.

16. SH Akbas, A Yegin, T Ozben (2005) Effect of pentylenetetrazol-induced epileptic seizure on the antioxidant enzyme activities, glutathione and lipid peroxidation levels in rat erythrocytes and liver tissues. Clin Biochem 38(11): 1009-1014.

17. Carmona Aparicio L, Pérez Cruz C, Zavala Tecuapetla C, et al. (2015) Overview of Nrf2 as therapeutic target in epilepsy. International Journal of Molecular Sciences 16(8): 18348-18367.
18. Mazzuferi M, Kumar G, van Eyll J, Danis B, Foerch P (2013) Nrf2 defense pathway: experimental evidence for its protective role in epilepsy. Annals of Neurology 74(4): 560-568.

19. Vargas M R, Johnson J A (2009) The Nrf2-ARE cytoprotective pathway in astrocytes. Expert Reviews in Molecular Medicine 11: e17.

20. Salim S (2017) Oxidative stress and the central nervous system. Journal of Pharmacology and Experimental Therapeutics 360(1): 201-205.

21. Saso L, Firuzi O (2014) Pharmacological applications of antioxidants: lights and shadows. Current Drug Targets 15(13): 1177-1199.

22. Manmohan KP, Purnima M, Maheshwari PK (2012) The lipid peroxidation product as a marker of oxidative stress in epilepsy. J Clin Diagn Res 6(4): 590-592. 